\title{
Construction and functional analysis of fatty acid desaturase gene disruptants in Candida albicans
}

\begin{abstract}
Correspondence
Somay Yamagata Murayama somay@lisci.kitasato-u.ac.jp
\end{abstract}

Received 8 December 2005

Revised 24 January 2006

Accepted 26 January 2006

\author{
Somay Yamagata Murayama, ${ }^{1}$ Yumiko Negishi, ${ }^{1,2,3}$ Takashi Umeyama, ${ }^{2}$ \\ Aki Kaneko, ${ }^{2}$ Takahiro Oura, ${ }^{3}$ Masakazu Niimi, ${ }^{2}$ Kimiko Ubukata $^{1}$ \\ and Susumu Kajiwara ${ }^{3}$
}

\begin{abstract}
${ }^{1}$ Laboratory of Infectious Agents Surveillance, Kitasato Institute for Life Sciences and Graduate School of Infection Control Sciences, Kitasato University, 5-9-1 Shirokane, Minato-ku, Tokyo 108-8641, Japan
${ }^{2}$ Department of Bioactive Molecules, National Institute of Infectious Diseases, 1-23-1 Toyama, Shinjuku-ku, Tokyo 162-8640, Japan
${ }^{3}$ Department of Life Science, Graduate School of Bioscience and Biotechnology, Tokyo Institute of Technology, 4259 Nagatsuta, Midori-ku, Yokohama, Kanagawa 226-8501, Japan

Polyunsaturated fatty acids (PUFAs), including linoleic acid (C18:2) and $\alpha$-linolenic acid (C18:3), are major components of membranes. PUFAs are produced from monounsaturated fatty acids by several fatty acid desaturases (FADs) in many fungi, but Saccharomyces cerevisiae, Schizosaccharomyces pombe and humans do not have these enzymes. Although the fungal pathogen Candida albicans produces C18:2 and C18:3, the enzymes that synthesize them have not yet been investigated. In this report, two ORFs, CaFAD2 and $\mathrm{CaFAD3}$, were identified based on their homology to other yeast FADs, and CaFAD2 and CaFAD3 gene disruptants were constructed. Cafad2 $\Delta$ and Cafad3 $\Delta$ lost their ability to produce C18:2 and C18:3, respectively. Furthermore, $S$. cerevisiae cells expressing CaFad2p converted palmitoleic acid (C16:1) and C18: 1 to hexadecadienoic acid (C16:2) and C18:2, respectively, and CaFad3p-expressing cells converted $\mathrm{C} 18: 2$ to $\mathrm{C} 18: 3$. These results strongly supported that $\mathrm{CaFAD2}$ encodes the $\triangle 12$ FAD and that $\mathrm{CaFAD} 3$ encodes the $\omega 3 \mathrm{FAD}$. However, phenotypic analysis demonstrated that the presence of these PUFAs did not affect the virulence to mice, or morphogenesis in the culture media used to induce morphological change of C. albicans.

\section{INTRODUCTION}

Fatty acids, whose principal function is to modulate the physical state of cell membranes, especially fluidity, are essential components for all organisms (Kitajima \& Nozawa, 1996). Various environmental factors, such as temperature and nutrition, lead organisms to modify their fatty acid composition and maintain their optimal membrane fluidity in order to adapt to new environments. Cells grown at a lower temperature contain a larger proportion of polyunsaturated fatty acids (PUFAs) in their total lipids, and the increase in fatty acid unsaturation is thought to compensate for the decreased membrane fluidity resulting from the lower environmental temperature (Prasad et al., 1996).

PUFAs, including linoleic acid (C18:2) and $\alpha$-linolenic acid (C18:3), are synthesized by a series of membrane-bound fatty acid desaturases (FADs) in eukaryotic cells. To begin with, the $\Delta 9$ FAD introduces a first double bond at the $\Delta 9$

Abbreviations: DMOX, 4,4-dimethyloxazoline; FAD, fatty acid desaturase; PUFA, polyunsaturated fatty acid; UFA, unsaturated fatty acid. position of both palmitic acid $(\mathrm{C} 16: 0)$ and stearic acid (C18:0), and converts them to monounsaturated fatty acids - palmitoleic acid $(\mathrm{C} 16: 1)$ and oleic acid $(\mathrm{C} 18: 1)$. The $\Delta 12$ FAD introduces a second double bond at the $\Delta 12$ position of $\mathrm{C} 16: 1$ and $\mathrm{C} 18: 1$, and converts them to hexadecadienoic acid $(\mathrm{C} 16: 2)$ and $\mathrm{C} 18: 2$. Additionally, the $\omega 3 \mathrm{FAD}$ introduces a third double bond at the $\Delta 15$ position of C18:2 and converts it to C18: 3 (Oura \& Kajiwara, 2004). Generally, deletion of $\triangle 9 \mathrm{FAD}$ is lethal due to the organism's inability to produce unsaturated fatty acids (UFAs).

In higher organisms, UFAs are known to play important roles as precursors of signalling molecules as well as structural molecules. PUFAs and their derivatives have been shown to act as developmental signals that induce asexual sporulation in some filamentous fungi (Calvo et al., 1999, 2001). Specifically, the $\Delta 12$ FAD mutant of the oilseed pathogen Aspergillus parasiticus has been reported to delay spore germination and to reduce conidia production (Wilson et al., 2004). However, Krishnamurthy et al. (2004) reported that a strain conditionally expressing $O L E 1$, which encodes $\triangle 9 \mathrm{FAD}$ in the major fungal pathogen 
Candida albicans, was incapable of producing UFAs, and was defective in hyphal development and chlamydospore formation under repressed conditions. This indicated the involvement of PUFAs in morphogenesis.

C. albicans, which is responsible for candidiasis, causes lifethreatening infection when host defences are impaired (Odds, 1987, 1988). The organism is a dimorphic yeast; it can exist as both yeast and filamentous mycelia. Both forms are pathogenic, but the mycelial forms penetrate tissues more easily and are, therefore, considered to be more pathogenic (Sobel et al., 1984). However, the pathogenic roles of morphological changes remain to be elucidated, because both morphological forms are found in infected tissues and because the molecular mechanisms of the dimorphic transition have not yet been understood clearly. Interestingly, the level of UFAs tended to be higher in the mycelial form than in the yeast form (Sadamori, 1987; Yano et al., 1982; Ghannoum et al., 1986). There is immense interest in studying lipid pathways for several reasons: there are a limited number of effective and safe systemic antifungal drugs, lipids play an important role in pathogenicity and morphogenesis, and lipids provide potential targets for a novel class of antifungals.

Despite their importance, the enzymes that catalyse the synthesis of PUFAs are not well understood compared to $\Delta 9$ FAD. This is partially because the model yeasts Saccharomyces cerevisiae and Schizosaccharomyces pombe do not possess these enzymes that are involved in the reactions following the one by $\Delta 9$ FAD (Mishra et al., 1992). In addition, only three enzymes that desaturate the $\Delta 9, \Delta 6$ and $\Delta 5$ positions are detected in humans, in whom C18:2 and C18:3 are essential fatty acids (Nakamura \& Nara, 2004).

In this study, we identified two genes in C. albicans that encode $\Delta 12$ and $\omega 3$ FADs, and named them CaFAD2 and $C a F A D 3$, respectively. Moreover, in an effort to elucidate the functions of these enzymes, we constructed $\mathrm{Cafad} 2 \Delta$ and Cafad $3 \Delta$ null mutants. However, our results showed that an alteration in the composition of fatty acids resulting from the deletion of CaFAD2 or CaFAD3 did not affect morphogenesis or virulence under the conditions studied.

\section{METHODS}

Strains, plasmids and growth conditions. The yeast strains used and generated in this work are listed in Table 1. We used Escherichia coli DH5 $\alpha$ and the cloning vector pBluescript II KS(-) (Stratagene) for DNA manipulation. The expression vector for $S$. cerevisiae, pYES2, was purchased from Invitrogen. The expression vector pFLAG-Actl (Umeyama et al., 2002) was used for gene expression in C. albicans. Yeast strains were grown routinely at $30^{\circ} \mathrm{C}$ in YPD medium $(1 \% \mathrm{w} / \mathrm{v}$ yeast extract, $2 \% \mathrm{w} / \mathrm{v}$ bactopeptone, $2 \% \mathrm{w} / \mathrm{v}$ glucose) or YNB medium. The YNB media consisted of the following: SD-ura $[0.67 \% \mathrm{w} / \mathrm{v}$ Difco yeast nitrogen base without amino acids, $2 \% \mathrm{w} / \mathrm{v}$ glucose, $0 \cdot 77 \mathrm{~g} \mathrm{l}^{-1}$ complete supplement mixture (CSM)-ura (Q-Biogene)], SD-arg-ura [having the same composition as SD-ura except with $0.72 \mathrm{~g} \mathrm{l}^{-1}$ CSM-arg-ura (Q-Biogene) instead of CSM-ura], SD-ura-raf (having the same composition as SD-ura supplemented with $2 \% \mathrm{w} / \mathrm{v}$ raffinose). The Candida strains $\left(\mathrm{Ura}^{-}\right.$) were grown in YPDU medium [YPD medium supplemented with uridine $(100 \mu \mathrm{g}$ $\mathrm{ml}^{-1}$ )]. Induction of filamentation in C. albicans was performed on the following solid media: Lee's medium (Lee et al., 1975), Spider medium (Liu et al., 1994) and serum medium (2\% w/v agar containing $10 \% \mathrm{v} / \mathrm{v}$ calf serum).

Cloning and DNA sequencing of $\boldsymbol{C}$. albicans FAD genes. The CaFAD2 coding sequence was amplified by PCR using the primers FAD2-5' (5'-GCCGGATCCAGGATGGCAGCTGCTACAACGTCC-3') and FAD2-3' (5'-GGCGCATGCTCAATGGGTTTGCTTTTTAGGATCGAC-3') (BamHI or SphI site is underlined), and then ligated into the EcoRV site of the cloning vector pBluescript II $\mathrm{KS}(-)$ (Stratagene), generating pBS-CaFAD2. The plasmid pBS-CaFAD3 was constructed in a similar way. The CaFAD3 ORF was amplified with the primers FAD3-5' (5'-GCCGGATCCAGGATGAGTGTAGTTGAGGCATCATCAAG-3') and FAD3-3' (5'-GGCGCATGCTTAATCTTTTGGTTTAACTGGTCC- $3^{\prime}$ ). Both strands were sequenced using a BigDye Terminator v3.1 Cycle Sequencing Kit (Applied Biosystems) on an ABI PRISM 3100-Avant genetic analyser (Applied Biosystems).

Gene disruption in C. albicans. Gene disruption was performed using a method similar to that described by Hanaoka et al. (2005). Briefly, for CaFAD2, two fragments, disFAD2-A and disFAD2-B, were amplified using primers disFAD2-1 (5'-GATAGATGACTTCTTTGGATGAAC- $\left.3^{\prime}\right)$ and disFAD2-2 (5'-GTCGTGACTGGGAAAACCCTGGCGAATAATTATTACAACTGATTGAAT-3'), and disFAD2-3

Table 1. Yeast strains

\begin{tabular}{|c|c|c|}
\hline Strain & Genotype & Source \\
\hline \multicolumn{3}{|c|}{ S. cerevisiae } \\
\hline IFO10150 & MATa ste-VC9 ura3-52 trp1-289 his3- $\Delta 1$ leu2-3,112 & $\mathrm{IFO}^{*}$ \\
\hline \multicolumn{3}{|l|}{ C. albicans } \\
\hline TUA4 & 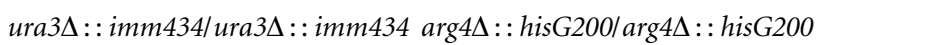 & Kaneko et al. (2004) \\
\hline TUA6 & 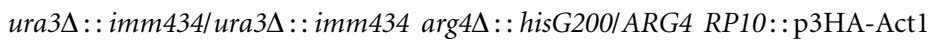 & Hanaoka et al. (2005) \\
\hline FAD2-2 & 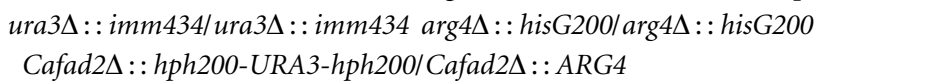 & This study \\
\hline FAD3-2 & 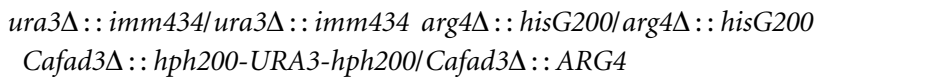 & This study \\
\hline
\end{tabular}

${ }^{\star}$ IFO, Institute for Fermentation, Osaka, Japan. 
(5' -CCTGTGTGAAATTGTTATCCGCTCTATTGATTGATTTACTTCTTTATG- ${ }^{\prime}$ ) and disFAD2-4 (5' ${ }^{\prime}$-TTCACTTGAACATATAGATAATAC$\left.3^{\prime}\right)$, respectively. Each fragment was used as a flanking homology region for a gene disruption cassette. For $C a F A D 3$, two fragments, disFAD3-A and disFAD3-B, were amplified using primers disFAD3-1 (5'-ATCAATAACAATAGAGATTTGTAC-3') and disFAD3-2 (5' GTCGTGACTGGGAAAACCCTGGCGTGCAAGATTCAATAACAAGAAATG-3'), and disFAD3-3 (5'-CCTGTGTGAAATTGTTATCCGCTCTATTGATTGATTTACTTCTTTATG-3') and disFAD3-4 (5'-TGTGGTTTTACCAGAGTCAATATG-3'), respectively. The PCRamplified disruption cassettes containing an hph200-URA3-hph200 or ARG4 marker were transformed into the TUA4 arg4 ura3 strain. Finally, both alleles of the CaFAD2 and CaFAD3 loci were replaced with $h p h 200$ and ARG4, yielding the respective strains FAD2-2 and FAD3-2.

Heterologous expression in S. cerevisiae. The fragment containing $C a F A D 2$ was isolated from pBS-CaFAD2, and ligated into the $B a m H I$ and SphI sites of the pYES2 expression vector (Invitrogen). The resulting pYES2-CaFAD2 was transformed into $S$. cerevisiae IFO10150 by the lithium acetate method (Burke et al., 2000). The transformants were cultivated in SD-ura-raf medium overnight at $30^{\circ} \mathrm{C}$. This overnight culture was transferred to fresh SD-ura-raf medium, and expression was induced by the addition of $1 / 100$ volume $20 \%(\mathrm{w} / \mathrm{v})$ galactose solution. The same strategy was used with SD-ura-raf medium supplemented with $1 \mathrm{mM} \mathrm{C18:2}$ for CaFAD3 expression.

Fatty acid analysis. Total lipids were extracted from exponentially growing cells $\left(\mathrm{OD}_{600} 1 \cdot 0-1 \cdot 5\right)$ with chloroform/methanol $(1: 2, \mathrm{v} / \mathrm{v})$ and were then methylated using $1 \%(\mathrm{v} / \mathrm{v})$ sulfuric acid in methanol, as described previously (Watanabe et al., 2004). The resulting fatty acid methyl esters (FAMEs) were extracted in hexane and analysed by GLC (GC-18A, Shimadzu) with a $0.25 \mathrm{~mm} \times 25 \mathrm{~m}$ HR-SS-10 capillary column (Shinwa Chemical Industries). A FAME mixture used as a fatty acid standard was purchased from Sigma. Fatty acid 4, 4-dimethyloxazoline (DMOX) derivatives were prepared as described previously (Oura \& Kajiwara, 2004). The derivatives were analysed by a GC mass spectrometer (QP5000; Shimadzu) operating at an ionization voltage of $70 \mathrm{eV}$, with a scan range of $40-400 \mathrm{kDa}$. The mass spectrum of any new peak obtained was interpreted by comparing it to that of the DMOX derivative of $\mathrm{C} 18: 2$ or $\mathrm{C} 18: 3$, prepared from $\mathrm{C} 18: 2$ or $\mathrm{C} 18: 3$ methyl ester (Sigma), and to the published value (reviewed by Spitzer, 1997).

Animal study. For each group, five male CD-1 (ICR) mice aged 4 weeks (Charles River Japan) weighing approximately $21-25$ g were inoculated with $10^{6}$ c.f.u. by intravenous injection. Survival curves were calculated using the Kaplan-Meier method and then were compared using the log-rank test. A $P$ value of less than 0.05 was considered significant.

\section{RESULTS}

\section{Cloning and sequencing of the CaFAD2 and CaFAD3 genes}

Based on the ORFs released in the Candida genome database website (http://www.candidagenome.org/), we detected two sequences having high homology with Saccharomyces kluyveri $\mathrm{FAD} 2$ and FAD3 proteins (SkFAD2p and $S k \mathrm{FAD} 3 \mathrm{p}$ ), respectively. The resulting two amino acid sequences, CaFAD2p (orf19-118) and CaFAD3p (orf19-4933), were predicted to be 437 and 434 aa polypeptides, respectively. The deduced amino acid sequence of $C a F A D 2 p$ had the highest identity with that of SkFAD2p $(63 \cdot 1 \%)$, and the amino acid sequences of $C a$ FAD3p and $S k F A D 3 p$ had $63.2 \%$ identity (Fig. 1 ). With respect to the three histidine cluster motifs that are conserved among the $\Delta 12$ and $\omega 3$ FADs, and that are essential for FAD activity as potential ligands for iron atoms (Los \& Murata, 1998), the motifs in both $C a F A D 2 p$ and $C a F A D 3 p$ are also highly conserved (Fig. 1).

\section{Gene disruption of CaFAD2 and CaFAD3}

To investigate the functions of the proteins encoded by the CaFAD2 and CaFAD3 genes, we constructed FAD2-2 (Cafad2 $\Delta$ mutant) and FAD3-2 (Cafad $3 \Delta$ mutant) strains of C. albicans, as described in Methods. Southern blotting and PCR amplification was used to confirm that the FAD2-2 and FAD3-2 mutants obtained were disrupted at the FAD2 and FAD3 loci, respectively, and the growth rate of each mutant at $30{ }^{\circ} \mathrm{C}$ in YPD medium was identical to that of the wild-type TUA6 strain (data not shown). The major fatty acids extracted from TUA6, FAD2-2 and FAD3-2 were determined by using GC analysis (Fig. 2a, b, c). No PUFA was detected in strain FAD2-2 (Fig. 2b), and C18:3 was not detected in strain FAD3-2 (Fig. 2c). The loss of PUFAs in each disruptant was restored by introducing a CaFAD2or CaFAD3-expressing plasmid (data not shown). These results suggest that the CaFAD2 gene was involved in $\mathrm{C} 16: 2$ and $\mathrm{C} 18: 2$ syntheses, and that the CaFAD3 gene was involved in $\mathrm{C} 18: 3$ synthesis.

\section{Heterologous expression of the CaFAD2 or CaFAD3 gene}

To examine whether or not $C a F a d 2 p$ functions as a $\Delta 12$ $\mathrm{FAD}$, the CaFAD2 gene was expressed in $S$. cerevisiae IFO10150. Fatty acids were extracted from the S. cerevisiae strain harbouring pYES2-CaFAD2 and analysed by GLC. The resultant data demonstrated two unique major peaks in the CaFAD2-expressing strain (Fig. 3b), which were absent in the strain harbouring only the vector (Fig. 3a). These peaks were suggested to be derived from $\mathrm{C} 16: 2$ and $\mathrm{C} 18: 2$, according to the retention times of C16:2 and C18:2 methyl esters ( 9.3 and $13.3 \mathrm{~min}$ ), respectively. Moreover, to determine the position at which the second double bond is induced, the GC-MS analysis of the fatty acid DMOX derivative suggested as C18:2 was performed. The mass spectrum of the fatty acid DMOX derivative exhibited a peak at $m / z=333$, and the peaks produced by ionization at $m / z=196,208,236$ and 248 were consistent with the double bonds at the $\Delta 9$ and $\Delta 12$ positions of the fatty acid (Fig. $4 \mathrm{a}$ ). These results demonstrate that the CaFAD2 gene encodes the $\Delta 12 \mathrm{FAD}$ in $C$. albicans, and that the $\mathrm{CaFad} 2 \mathrm{p}$ enables the $S$. cerevisiae cells to produce $\mathrm{C} 16: 2$ and $\mathrm{C} 18: 2$.

Next, we examined whether or not $\mathrm{CaFad} 3 \mathrm{p}$ functions as an $\omega 3 \mathrm{FAD}$ in a similar way. The plasmid for CaFAD3 gene expression was introduced into S. cerevisiae IFO10150 and the expression of this gene was then induced in the presence of C18:2 as a substrate. The GC analysis of fatty acids 


\begin{tabular}{|c|c|c|c|}
\hline CaFAD2 & 1 & MAAATTSFSSGFNNNNNADQSTDSSATISKSGNVASFKTTSTTSTYQTNLTAIDTYGNEFKVPDYTIKDILSAIP & 75 \\
\hline$S k F A D 2$ & 1 & 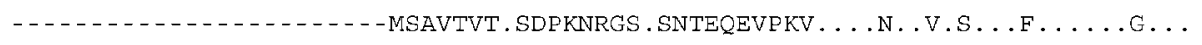 & 51 \\
\hline $\mathrm{CaFAD} 3$ & 1 & 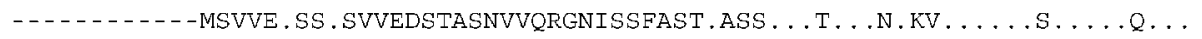 & 63 \\
\hline SkFAD3 & 1 & 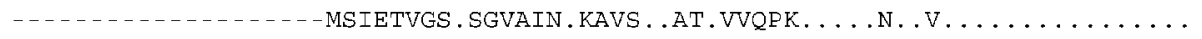 & 55 \\
\hline CaFAD2 & 76 & THCYERRLIQSLSYVFRDIFCMVVLGFI ANNY IHLI - - - - PN-QF - IRFAAWTGYVWCQGLFGTGIWVLAHECG & 143 \\
\hline$S k F A D 2$ & 52 & 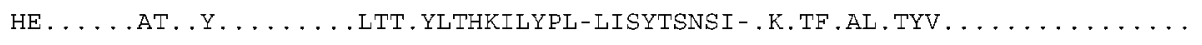 & 124 \\
\hline CaFAD3 & 64 & 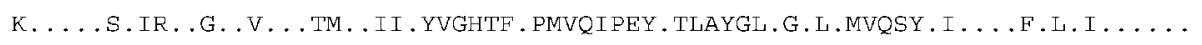 & 138 \\
\hline SkFAD3 & 56 & 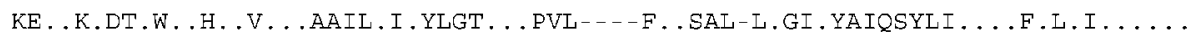 & 125 \\
\hline CaFAD2 & 144 & HOAFSDYGSVNDFVGWVLHSYLLVPYFSWKF SHGKHHKATGHLTRDMVFVPKTKEEFLONRGV-KDLDDLLGDSP & 217 \\
\hline SkFAD2 & 125 & 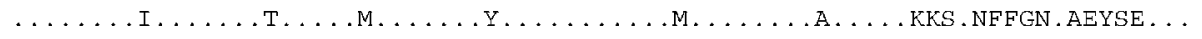 & 199 \\
\hline CaFAD3 & 139 & 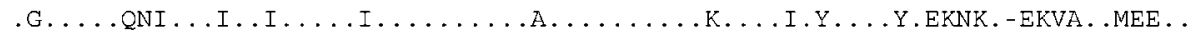 & 212 \\
\hline SkFAD3 & 126 & 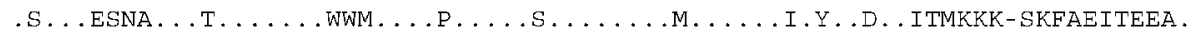 & 199 \\
\hline CaFAD2 & 218 & MYSLLTLIFQQTFGWISYLVANVSGQKYPGVSF LKLNHFNPNSLIFDKKDYWY ILLSDLGILLQFFNLYVWYQSF & 292 \\
\hline SkFAD2 & 200 & 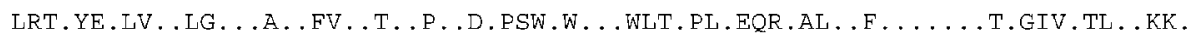 & 274 \\
\hline CaFAD3 & 213 & I . F.V.V. . LG.LQL . AT.AT. .V . .Y.KIAKS.YT.T.PV . . HQ . . V. . I . I. A.TTV.Q . KN. & 287 \\
\hline SkFAD 3 & 200 & VMT . FN . A . . VG. LQL . AT . AT . . P . . . KKFFKS. YW. T. PV . A . .F.W. IM . I . .VSTLLIN . L . RAY & 274 \\
\hline CaFAD2 & 293 & GGFNLLVNYVL PYFLVNHWLVF I TYLQHSDPQMPHYEASQWTFARGAAATIDREFGFVGKHIFHDI IETHVLHHY & 367 \\
\hline SkFAD2 & 275 & 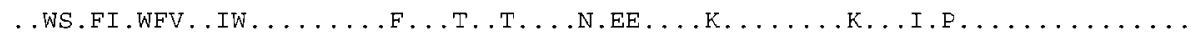 & 349 \\
\hline CaFAD3 & 288 & 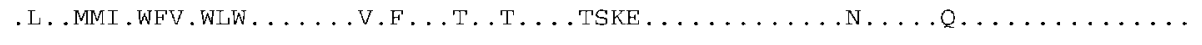 & 362 \\
\hline SkFAD3 & 275 & 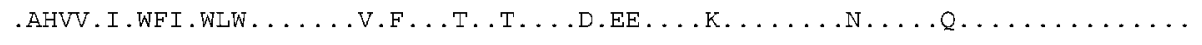 & 349 \\
\hline $\mathrm{CaFAD} 2$ & 368 & VSRI PFYNAREASEAI KKVMGIHYQHSDENMWVSLWKSARWCQFVDGN- - - -NGVLMYRNTNGFGVDFKKQTH- - & 436 \\
\hline$S k F A D 2$ & 350 & 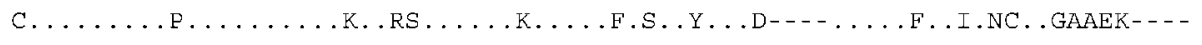 & 416 \\
\hline $\mathrm{CaFAD} 3$ & 363 & 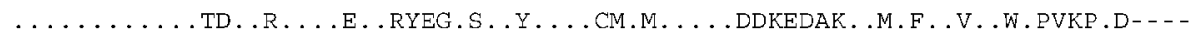 & 433 \\
\hline SkFAD3 & 350 & 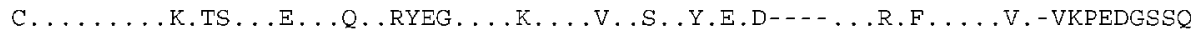 & 419 \\
\hline
\end{tabular}

Fig. 1. Comparison of the deduced amino acid sequences of Fad2p and Fad3p between C. albicans and S. kluyveri. Underlining indicates the histidine cluster motif. Sequences were aligned using the FASTA algorithm. CaFAD2 is used as the leading strand. Dots indicate amino acids identical to this reference sequence and alignment gaps are denoted by hyphens.

(a)

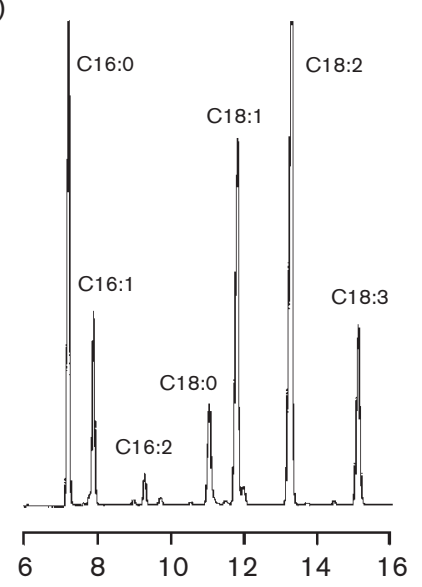

(b)

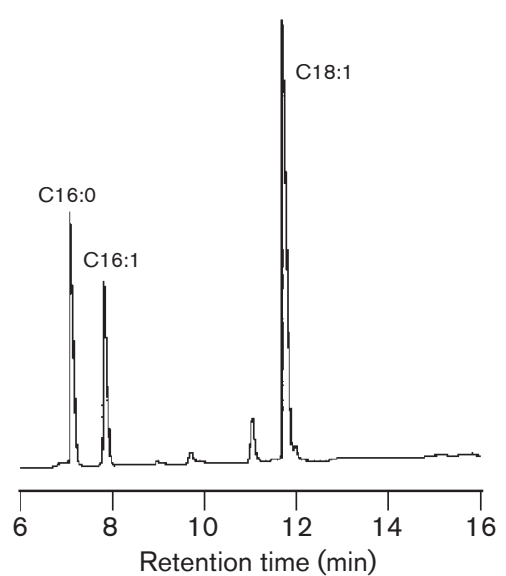

(c)

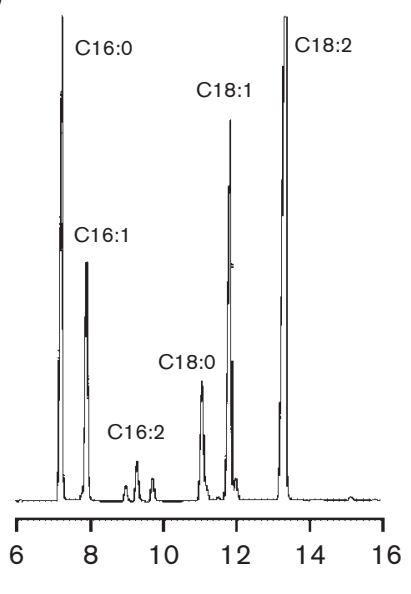

Fig. 2. GC analysis of fatty acids in total lipids extracted from C. albicans strains. The wild-type strain TUA6 (a), the Cafad2 $\Delta$ disruptant (b) and the Cafad3 $\Delta$ disruptant (c) were cultured in SD-arg-ura medium at $30{ }^{\circ} \mathrm{C}$. C16:0, palmitic acid; C16:1, palmitoleic acid; C16:2, hexadecadienoic acid; C18:0, stearic acid; C18:1, oleic acid; C18:2, linoleic acid; C18:3, $\alpha$-linolenic acid. 
(a)

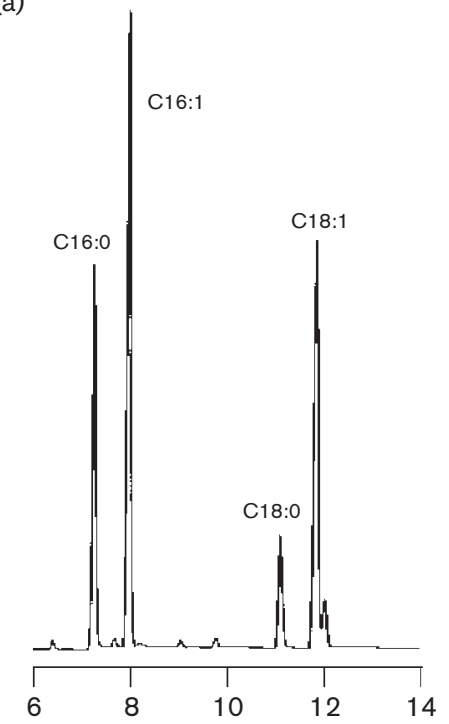

(c)

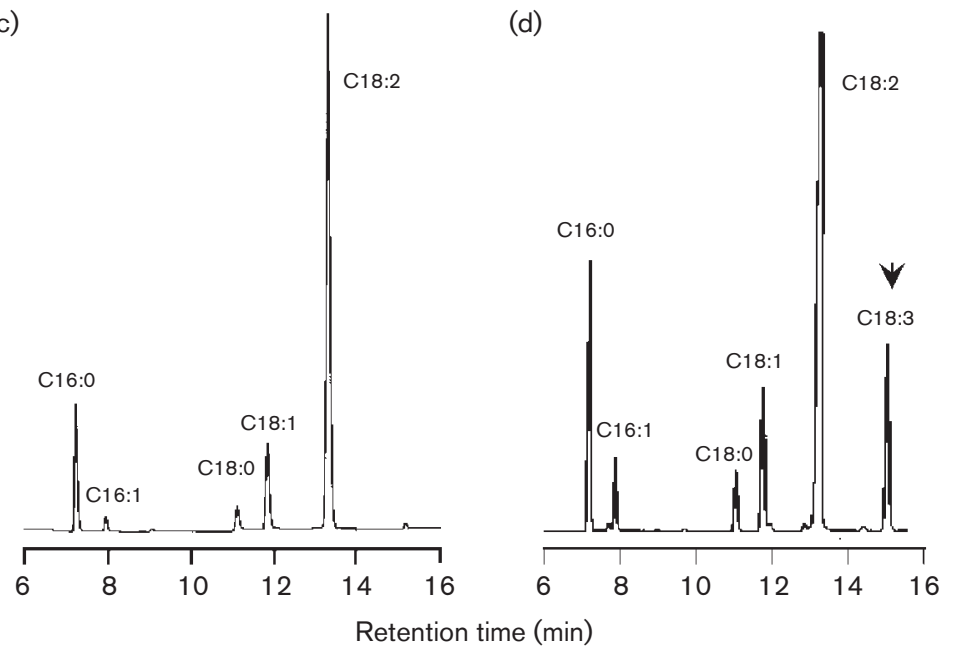

(b)

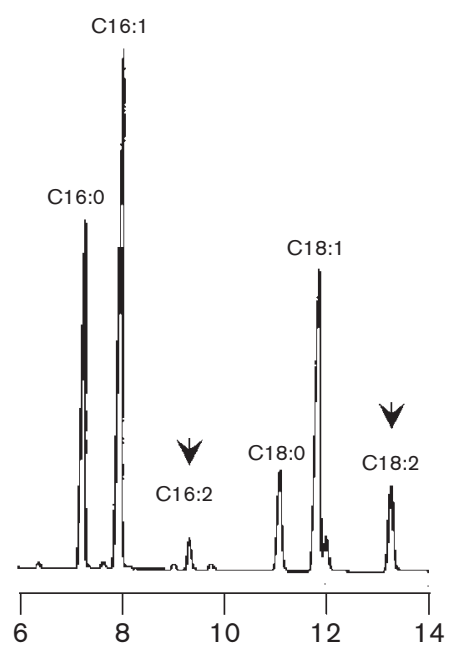

Fig. 3. GC analysis of fatty acids in total lipids extracted from $S$. cerevisiae transformants. The S. cerevisiae IFO10150 strain harbouring the pYES2 vector (a) or pYES2CaFAD2 (b) was cultured in SD-ura-raf medium, and the $S$. cerevisiae strain harbouring the pYES2 vector (c) or pYES2CaFAD3 (d) was cultured in SD-ura-raf medium supplemented with $1 \mathrm{mM} \mathrm{C18:2}$ at $30{ }^{\circ} \mathrm{C}$. A $1 / 100$ volume $20 \%(\mathrm{w} / \mathrm{v})$ galactose solution was added to each culture to induce protein expression. Arrowheads in (b) and (d) indicate the peaks specific to the $\mathrm{CaFADs}$-expressing strains and was analysed by GC-MS (Fig. 4). C16:0, palmitic acid; C16:1, palmitoleic acid; C16:2, hexadecadienoic acid; C18:0, stearic acid; C18:1, oleic acid; C18:2, linoleic acid; C18:3, $\alpha$-linolenic acid. extracted from the $S$. cerevisiae strain harbouring pYES2$\mathrm{CaFAD} 3$ exhibited an additional unique peak (Fig. 3d), which was absent when pYES2 was introduced in $S$. cerevisiae (Fig. 3c). This peak was derived from $\mathrm{C} 18: 3$, according to the retention time $(15 \cdot 1 \mathrm{~min})$ of its methyl ester (Fig. 3d), the mass spectrum $(\mathrm{m} / z=331)$ of its DMOX derivative, and the cleavage peaks at $m / z=196,208,236$, 248, 276 and 288 (Fig. 4b), which were consistent with the double bonds at the $\Delta 9, \Delta 12$ and $\Delta 15$ positions of the fatty acid. These results revealed that the CaFAD 3 gene encodes an $\omega 3$ FAD in $C$. albicans and that the $C a F A D 3$ protein confers ability to produce $\mathrm{C} 18: 3$ on $S$. cerevisiae.

\section{Phenotypic analysis of Cafad2 $\Delta$ and Cafad3A disruptants}

To examine the cold-sensitivity of the mutants we examined the growth rate of both Cafad $2 \Delta$ and $C a f a d 3 \Delta$, and the wild-type strain TUA6, at 10,15 and $20^{\circ} \mathrm{C}$ in YPD media.
The experiment was done twice. There was no significant difference in the $\mathrm{OD}_{610}$ and c.f.u. among strains. To investigate the effects of PUFAs on morphogenesis in C. albicans, we observed the hyphal formation of both Cafad $2 \Delta$ and Cafad $3 \Delta$ mutants on solid media or in liquid media, such as Lee's medium, Spider medium and serum medium, at 25, 30 and $37^{\circ} \mathrm{C}$. The disruptant strains grew equally as well as the wild-type strain TUA6 under these conditions, and the hyphal formation of the disruptants was almost the same as that of TUA6 in all three media at all temperatures (data not shown). We also examined chlamydospore formation, an alternative morphogenetic event in C. albicans. For chlamydospore formation, cells were streaked on cornmeal agar with $1 \%$ (v/v) Tween 80 (Buckley et al., 1982), covered with coverslips to maintain a semi-aerobic environment and incubated at $25^{\circ} \mathrm{C}$ for 7 days. The disruptant strains formed chlamydospores normally (data not shown), indicating that PUFAs do not contribute to the formation of hyphae or chlamydospores. 


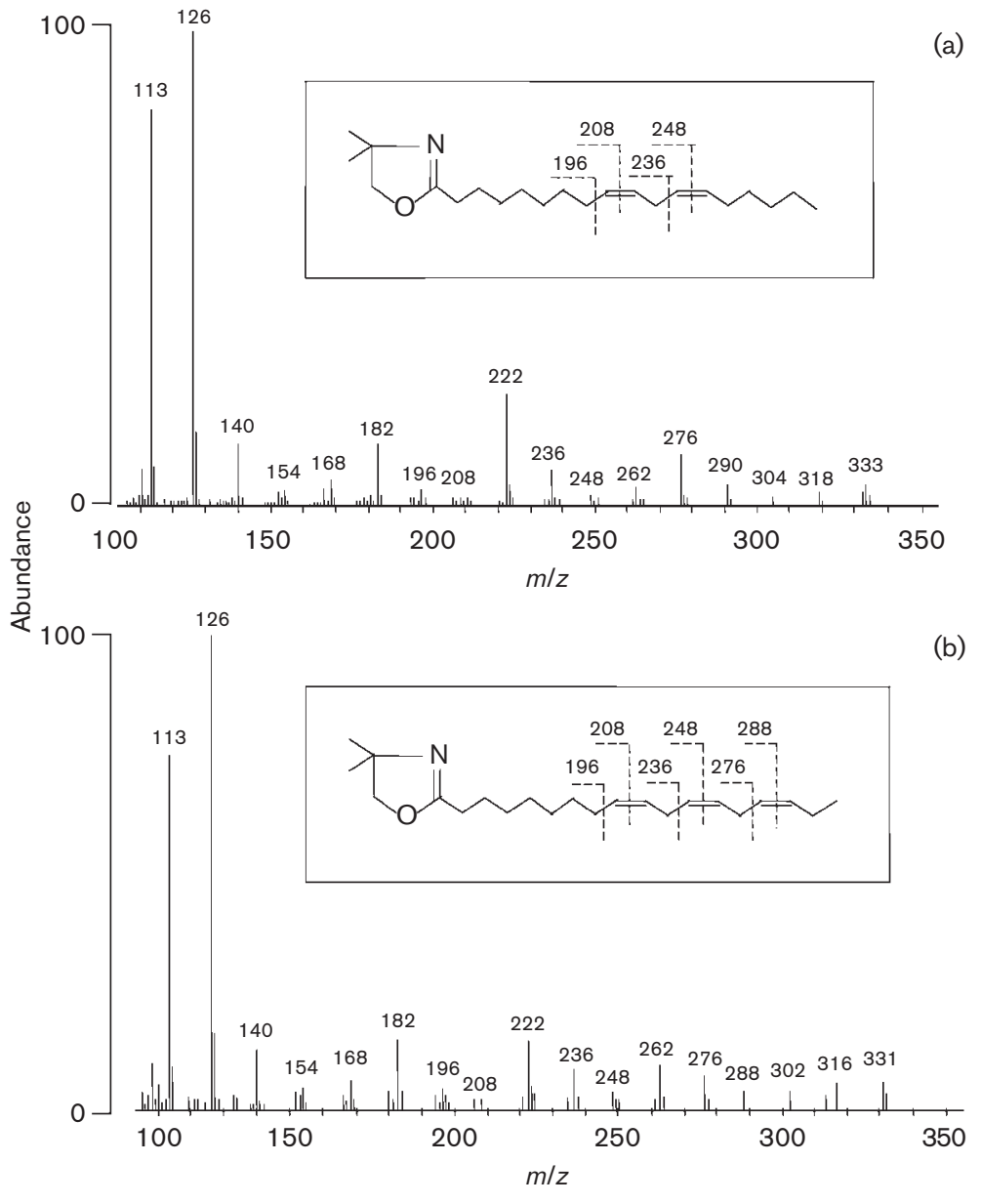

Fig. 4. GC-MS analysis of the DMOX derivative. The spectra of the DMOX derivative of the fatty acids indicated in Fig. 3. (a) linoleic acid derived from $S$. cerevisiae strain harbouring pYES2-CaFAD2 and (b) $\alpha$-linolenic acid derived from the strain harbouring pYES2CaFAD3. The deduced structure of the fatty acid derivative is shown above the spectrum.
To determine the role of PUFAs in virulence, mice were intravenously injected with $10^{6}$ c.f.u. of the wild-type

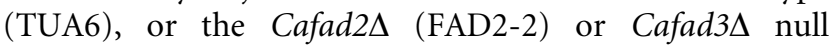
(FAD3-2) mutants, and monitored for survival. No significant difference in mortality was found (FAD2-2 vs TUA6, $P=0.73$; FAD3-2 vs TUA6, $P=0.31$ ), suggesting that PUFAs were not associated with the systemic infection with C. albicans.

\section{DISCUSSION}

This is believed to be the first report on the cloning and characterization of $\Delta 12$ and $\omega 3$ FADs in the dimorphic fungus $C$. albicans. $C a \mathrm{Fad} 2 \mathrm{p}$ was found to share $63 \cdot 1,55 \cdot 7$ and $54 \cdot 7 \%$ identity to $S k$ FAD 2 p, the $\Delta 12$ FAD of Aspergillus nidulans and the $\triangle 12 \mathrm{FAD}$ of $A$. parasiticus, respectively; and $C a \mathrm{Fad} 3 \mathrm{p}$ was found to share $63 \cdot 2 \%$ similarity to $S k F A D 3 p$. However, $C a F A D 2 p$ showed $59 \cdot 3$ and $56.3 \%$ identities with $C a F A D 3 p$ and $S k F A D 3 p$, respectively, percentages that are higher than those of the $\Delta 12$ FAD of the other fungi. Therefore, it was difficult to decide whether each $C a F A D$ gene is a $\triangle 12 \mathrm{FAD}$ or an $\omega 3 \mathrm{FAD}$. The high homology among such FADs in some yeasts and other organisms has fascinated us, impelling our investigation of the enzymes of $C$. albicans. We have provided biochemical data indicating that
$C a$ Fad $2 p$ or $C a$ Fad $3 p$ should function as only a $\Delta 12$ or $\omega 3$ FAD, respectively. However, we were unable to find any supporting evidence that PUFAs have some involvement in cold-sensitivity, morphogenesis in the media or virulence.

In some Aspergillus species, PUFAs and their derivatives act as developmental signals. For example, the mutants deleted of $\Delta 12$ FAD show different physiological phenomena compared to the wild-type (Wilson et al., 2004). Since similar functions of PUFAs are also expected in C. albicans, we disrupted the CaFAD2 and CaFAD3 genes. However, phenotypic characteristics such as germ tube formation, hyphal morphogenesis and chlamydospore formation (Nobile et al., 2003) did not differ among the wild-type strain, Cafad2A and $C a f a d 3 \Delta$ (data not shown), although it was reported that the C. albicans $\triangle 9$ FAD gene (OLE1) affects viability and morphogenesis significantly (Krishnamurthy et al., 2004). There may be small amounts of fatty acids in some media containing serum, which might complement the hypothesized defects involving PUFAs. But we were able to exclude this hypothesis by using a hyphae-inducing synthetic medium such as Spider medium. These facts suggested that C18 : 1, not PUFAs, plays a key role in morphogenesis or in signalling for the morphogenetic transition in C. albicans, unlike the case with Aspergillus species. In fact, some yeasts, 
like S. cerevisiae and Schizosaccharomyces pombe, do not have FADs other than $\triangle 9 \mathrm{FAD}$, and do not require PUFAs for vegetative growth (Ratledge \& Evans, 1991).

In infected tissues, the mycelial form of $C$. albicans predominates. The major differences in the lipid composition between the two morphological forms, yeast and hyphae, are alterations in phospholipids and fatty acid composition (Yano et al., 1982). The transition into the hyphal form causes a great increase in linoleic acid, contained in all phospholipids, the presence of which accompanies a compensatory decrease in monounsaturated fatty acids. These alterations point to a higher degree of unsaturation. Unexpectedly, in our study there were no obvious differences in virulence to mice between the wild-type strain and the Cafad $2 \Delta$ or Cafad $3 \Delta$ disruptants. However, the mice were fed bait including fatty acids, and C. albicans is able to assimilate fatty acids from the cells of mice. Thus, in the future, animal studies using the C. albicans mutant that is unable to assimilate exogenous fatty acids are necessary in order to clarify the relationship between PUFAs and the virulence of this yeast.

The expression levels of FAD3 and FAD2 in S. kluyveri increased at low temperatures (Watanabe et al., 2004, Oura \& Kajiwara, 2004). In C. albicans, the proportion of PUFAs at $25^{\circ} \mathrm{C}$ has been shown to exceed that at $37^{\circ} \mathrm{C}$ (Brondz \& Olsen, 1990), suggesting the $\mathrm{CaFAD} 2$ and $\mathrm{CaFAD} 3$ enzymes might be involved in membrane fluidity or some other functions. Generally, higher temperatures increase membrane fluidity. The functional state of the lipid bilayer is a liquid-crystalline phase, but a decrease in temperature induces a gel-phase transition and a drastic loss of membrane properties. This phase transition essentially depends on the lipid composition of the membrane, especially the fatty acyl chains. The cis-unsaturated double bond in the chain induces a $30^{\circ}$ bend, which creates a cavity in the lipid layer and perturbs the packing density (Feller \& Gerday, 2003). Further studies under stressed growth conditions, such as at low temperatures or limited nutrition, e.g. in the human body, may unveil the hidden roles of FADs in $C$. albicans.

\section{ACKNOWLEDGEMENTS}

We thank Nozomu Hanaoka for technical assistance. A. Kaneko is supported by the Cooperative System for Supporting Priority Research, Japan Science and Technology Corporation (JST). This study was supported in part by a grant from the 21st Century COE Program, Ministry of Education, Culture, Sports, Science and Technology (MEXT), and by the Ministry of Education, Science, Sports and Culture, Grant-in-Aid for Scientific Research (C), 16590295, 2005.

\section{REFERENCES}

Brondz, I. \& Olsen, I. (1990). Fatty acid contents of the yeast and mycelial phase of Candida albicans. J Chromatogr 533, 152-158.

Buckley, H. R., Price, M. R. \& Daneo-Moore, L. (1982). Isolation of a variant of Candida albicans. Infect Immun 37, 1209-1217.
Burke, D., Dawson, D. \& Stearns, T. (2000). Methods in Yeast Genetics: a Cold Spring Harbor Laboratory Course Manual, 2000 edn. Cold Spring Harbor, NY: Cold Spring Harbor Laboratory.

Calvo, A. M., Hinze, L. L., Gardner, H. W. \& Keller, N. P. (1999). Sporogenic effect of polyunsaturated fatty acids on development of Aspergillus spp. Appl Environ Microbiol 65, 3668-3673.

Calvo, A. M., Gardner, H. W. \& Keller, N. P. (2001). Genetic connection between fatty acid metabolism and sporulation in Aspergillus nidulans. J Biol Chem 276, 25766-25774.

Feller, G. \& Gerday, C. (2003). Psychrophilic enzymes: hot topics in cold adaptation. Nat Rev Microbiol 1, 200-208.

Ghannoum, M. A., Khamis, J. L. \& Radwan, S. S. (1986). Dimorphism-associated variations in the lipid composition of Candida albicans. J Gen Microbiol 132, 2367-2375.

Hanaoka, N., Umeyama, T., Ueno, K., Ueda, K., Beppu, T., Fugo, H., Uehara, Y. \& Niimi, M. (2005). A putative dual-specific protein phosphatase encoded by YVH1 controls growth, filamentation and virulence in Candida albicans. Microbiology 151, 2223-2232.

Kaneko, A., Umeyama, T., Hanaoka, N., Monk, B. C., Uehara, Y. \& Niimi, M. (2004). Tandem affinity purification of the Candida albicans septin protein complex. Yeast 21, 1025-1033.

Kitajima, Y. \& Nozawa, Y. (1996). Lipids and dimorphism of Candida albicans and Sporothrix schenckii. In Lipids of Pathogenic Fungi, pp. 219-234. Edited by R. Prasad \& M. A. Ghannoum. Boca Raton, FL: CRC Press.

Krishnamurthy, S., Plaine, A., Albert, J., Prasad, T., Prasad, R. \& Ernst, J. F. (2004). Dosage-dependent functions of fatty acid desaturase Olelp in growth and morphogenesis of Candida albicans. Microbiology 150, 1991-2003.

Lee, K. L., Buckley, H. R. \& Campbell, C. C. (1975). An amino acid liquid synthetic medium for the development of mycelial and yeast forms of Candida albicans. Sabouraudia 13, 148-153.

Liu, H., Kohler, J. \& Fink, G. R. (1994). Suppression of hyphal formation in Candida albicans by mutation of a STE12 homolog. Science 266, 1723-1726.

Los, D. A. \& Murata, N. (1998). Structure and expression of fatty acid desaturase. Biochim Biophys Acta 1394, 3-15.

Mishra, P., Bolard, J. \& Prasad, R. (1992). Emerging role of lipids of Candida albicans, a pathogenic dimorphic yeast. Biochim Biophys Acta 1127, 1-14.

Nakamura, M. T. \& Nara, T. Y. (2004). Structure, function, and dietary regulation of $\Delta 6, \Delta 5$, and $\Delta 9$ desaturases. Annu Rev Nutrition 24, 345-376.

Nobile, C. J., Bruno, V. M., Richard, M. L., Davis, D. A. \& Mitchell, A. P. (2003). Genetic control of chlamydospore formation in Candida albicans. Microbiology 149, 3629-3637.

Odds, F. C. (1987). Candida infections: an overview. Crit Rev Microbiol 15, 1-5.

Odds, F. C. (1988). Factors that predispose the host to candidosis. In Candida and Candidosis, pp. 93-114. London: Baillière Tindall.

Oura, T. \& Kajiwara, S. (2004). Saccharomyces kluyveri FAD3 encodes an $\omega 3$ fatty acid desaturase. Microbiology 150, 1983-1990.

Prasad, R., Koul, A., Mukherjee, P. K. \& Ghannoum, M. A. (1996). Lipids of Candida albicans. In Lipids of Pathogenic Fungi, pp.105-138. Edited by R. Prasad \& A. M. Ghannoum. Boca Raton, FL: CRC Press.

Ratledge, C. \& Evans, C. T. (1991). Lipids and their metabolism. In The Yeast, 2nd edn, pp. 367-455. Edited by A. H. Rose \& J. S. Harrison. London: Academic Press.

Sadamori, S. (1987). Comparative study of lipid composition of Candida albicans in the yeast and mycelial forms. Hiroshima J Med Sci 36, 53-59. 
Sobel, J. D., Muller, G. \& Buckley, H. R. (1984). Critical role of germ tube formation in the pathogenesis of candidal vaginitis. Infect Immun 44, 576-580.

Spitzer, V. (1997). Structure analysis of fatty acids by gas chromatography - low resolution electron impact mass spectrometry of their 4,4-dimethyloxazoline derivatives - a review. Prog Lipid Res 35, 387-408.

Umeyama, T., Nagai, Y., Niimi, M. \& Uehara, Y. (2002). Construction of FLAG tagging vectors for Candida albicans. Yeast 19, 611-618.
Watanabe, K., Oura, T., Sakai, H. \& Kajiwara, S. (2004). Yeast $\Delta 12$ fatty acid desaturase: gene cloning, expression and function. Biosci Biotechnol Biochem 68, 721-727.

Wilson, R. A., Calvo, A. M., Chang, P.-K. \& Keller, N. P. (2004). Characterization of the Aspergillus parasiticus $\Delta^{12}$-desaturase gene: a role for lipid metabolism in the Aspergillus-seed interaction. Microbiology 150, 2881-2888.

Yano, K., Yamada, T., Banno, Y., Sekiya, T. \& Nozawa, Y.(1982). Modification of lipid composition in a dimorphic fungus, Candida albicans, during the yeast cell to hypha transformation. Jpn J Med Mycol 23, 159-165. 